\title{
Persistência de Veia Cava Superior Esquerda em Paciente Submetido a Implante de Marcapasso
}

\author{
Persistence of Left Superior Vena Cava in Patient submitted a Pacemaker \\ Implantation
}

Sara Carneiro Vicente Bueno ${ }^{1, *}$, Arnaldo Duarte Lourenço ${ }^{1}$

ORCID IDs

Bueno SCV (D) https://orcid.org/0000-0001-6638-2492

Lourenço AD (D) https://orcid.org/0000-0003-3582-6534

\section{RESUMO}

\begin{abstract}
A persistência da veia cava superior esquerda (PCVSE) é a malformação venosa torácica mais frequente, porém rara e com diagnóstico habitualmente acidental. Recorrentemente, sua maior suspeita é realizada no ato intraoperatório relacionado a uma dificuldade técnica na realização do procedimento, mesmo por profissionais mais experientes. Relato de caso: Paciente de 77 anos, sexo feminino, chagásica, submetida a implante de marcapasso definitivo, sendo o diagnóstico de persistência de veia cava superior esquerda realizado durante o procedimento cirúrgico. A paciente não apresentou intercorrência durante o procedimento e evoluiu com melhora clínica substancial após o implante do dispositivo cardíaco eletrônico, mantendo-se assintomática.
\end{abstract}

PALAVRAS-CHAVE: Veia cava superior; Malformações vasculares; Marcapasso artificial.

\begin{abstract}
The persistence of the left superior vena cava (PLSVC) is the most frequent thoracic venous malformation, however rare, and with a usually accidental diagnosis. Recurringly, its greatest suspicion is realized in the intraoperative act related to a technical difficulty in performing the procedure, even by more experienced professionals. Case report: patient is 77 years old, woman, chagasic, submitted a definitive pacemaker implant, being the diagnosis of persistence left superior vena cava realized during the surgical procedure. The patient did not present intercurrence during the procedure and developed with substantial clinical improvement after the implantation of the electronic cardiac device, and remaining asymptomatic.
\end{abstract}

KEYWORDS: Superior vena cava; Vascular malformations; Artificial pacemaker.

1. Hospital Ana Costa - Setor de Estimulação Cardíaca Artificial - Santos/SP - Brasil.

*Autor correspondente: sara.cvicente@gmail.com

Recebido: 16 Maio 2018 | Aceito: 04 Fev 2019

Editor Associado: J. Tarcísio Medeiros de Vasconcelos 


\section{INTRODUÇÃO}

Inicialmente descrita na década de 1950, a persistência da veia cava superior esquerda (PVCSE) é a anomalia vascular venosa mais encontrada no tórax, apesar de sua raridade ${ }^{1}$. Sua prevalência encontra-se entre 0,3 e 0,5\% da população mundial, contudo, quando associada a cardiopatias congênitas, sobe para 2,8 a 4,3\% $\%^{1-3}$. Na maioria dos casos, a veia cava superior direita está presente, sendo raro o achado de PVCSE isolada ${ }^{4}$.

Durante a fase embriológica, a drenagem é feita pelas veias cardinais: cabeça, pescoço e braços drenam para o átrio direito através das veias cardinais anteriores direita e esquerda ${ }^{1}$. Ao redor da oitava semana de gestação, a veia inominada esquerda une-se às porções anteriores das veias cardinais. A veia cardinal anterior direita torna-se a veia cava superior direita ${ }^{1-3}$. Já a veia cardinal anterior esquerda é ocluída, originando o ligamento de Marshall. ${ }^{1}$ Quando essa veia não se degenera, desenvolve-se a veia cava superior esquerda, a qual drenará para o átrio direito através do seio coronariano ${ }^{1,3}$. A PVCSE é uma condição embriológica que não traz maiores complicações ou sintomas, sendo geralmente identificada durante o implante de dispositivos cardíacos, catéteres venosos centrais ou procedimentos cirúrgicos torácicos ${ }^{1,4}$. Torna-se, então, um eventual obstáculo, mesmo aos profissionais experientes ${ }^{1,5}$

Apesar da sua benignidade relacionada à sintomatologia, essa anomalia torna o paciente mais vulnerável ao desenvolvimento de arritmias cardíacas, especialmente por alterações no nó atrioventricular e no feixe de $\mathrm{His}^{4,6}$. Em menor parte dos casos, essa condição pode obstruir parcialmente a válvula mitral, gerando um prejuízo no fluxo atrioventricular esquerdo, ocorrendo, então, a possibilidade do desenvolvimento de sintomas pelo paciente ${ }^{4}$.

Sua presença pode estar associada a outras anormalidades cardiovasculares, como defeito do septo atrial, válvula aórtica bicúspide, coarctação da aorta e atresia ostial do seio coronariano ${ }^{5}$.

\section{RELATO DE CASO}

Paciente do sexo feminino, 77 anos, com antecedente pessoal de doença de Chagas e insuficiência cardíaca, em tratamento otimizado, é internada com quadro de síncope relacionada à posição ortostática de início recente associada à dispneia e edema de membros inferiores. Ao eletrocardiograma, apresentava-se em ritmo sinusal com evidência de bloqueio de ramo direito. Durante a internação, realizou-se um ecocardiograma transtorácico (ECO 2D) que mostrou aumento importante do volume do átrio direito, com déficit contrátil importante do ventrículo esquerdo e leve do ventrículo direito, à custa de hipocinesia difusa de ambos e dilatação do seio coronariano. Ao longo do exame, a paciente permaneceu em vigência de arritmia. Optou-se pela realização de holter de 24 horas, três canais, no qual apresentou frequência cardíaca média de 50 batimentos por minuto em ritmo sinusal, bloqueio de ramo direito e bloqueio atrioventricular total durante o período do exame. Após avaliação, definiu-se que a paciente seria submetida a implante de marcapasso permanente. Durante o procedimento, já na passagem dos fios-guia pela punção da veia subclávia direita, foi feito o diagnóstico da PVCSE, pelo trajeto que apresentaram, descendo pela esquerda, e houve dificuldade na passagem do cabo-eletrodo ventricular, denotando-se a grande dilatação do seio coronariano apresentada ao ECO 2D. $\mathrm{O}$ procedimento terminou sem intercorrência, com o posicionamento adequado dos eletrodos atrial e ventricular. A paciente permaneceu internada para avaliação clínica pós-cirúrgica, evoluindo sem complicações. Recebeu alta hospitalar, mantendo-se em acompanhamento cardiológico regular, encontrando-se assintomática, com retomada completa da sua rotina habitual.

\section{DISCUSSÃO}

A suspeição da PVCSE ocorre, em geral, no momento intraoperatório, no entanto, sua existência também pode ser aventada por meio de exames ${ }^{4}$. À radiografia de tórax, nota-se um contorno anormal do mediastino superior à esquerda, com presença de uma proeminência abaixo do arco aórtico e ausência da linha de retificação da veia cava superior direita ${ }^{1,3}$ (Fig. 1).

Ao ECO 2D, pode-se encontrar a dilatação do seio coronariano, nesse caso mensurada em $2,2 \mathrm{~cm}$, corroborando o diagnóstico ${ }^{6}$. Nesse exame, confirma-se a presença da PVCSE por meio da injeção de solução salina agitada no membro superior esquerdo, demonstrando a ocorrência precoce de bolhas no seio coronariano, subsequentemente drenadas ao átrio direito ${ }^{1,5}$ (Fig. 2). A 


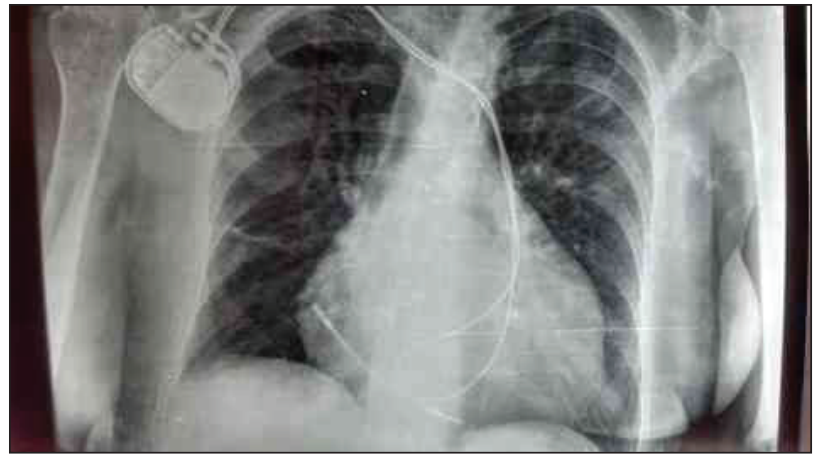

Figura 1. Raio $X$ de tórax demonstrando o posicionamento dos cabos-eletrodos atrial e ventricular na conclusão do procedimento.

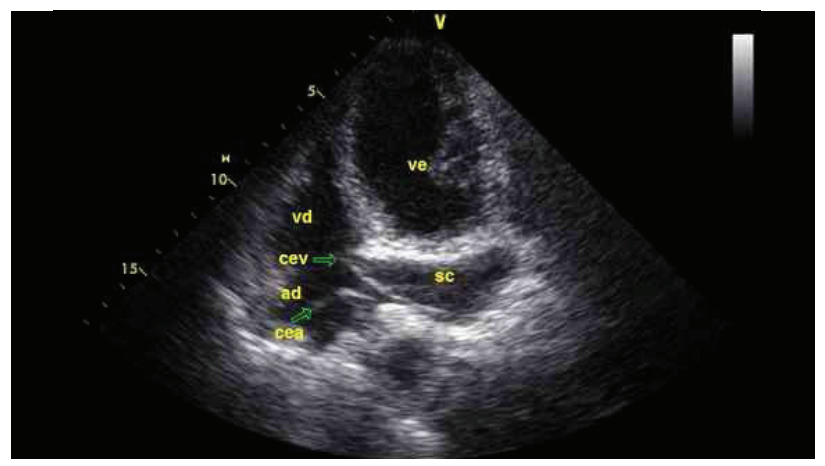

Figura 2. Ecocardiograma bidimensional demonstrando as relações do aumento do átrio direito e seio coronariano $(2,2 \mathrm{~cm}) \mathrm{com}$ a passagem e posicionamento dos cabos-eletrodos em átrio direito e ventrículo direito.

tomografia computadorizada e a ressonância magnética podem confirmar o diagnóstico da PVCSE, avaliando com maiores detalhes a anatomia da região ${ }^{1}$.

O paciente com PVCSE apresenta-se como desafio no posicionamento de dispositivos: inicialmente, pois não se conhece o diagnóstico, sendo o implante do dispositivo diferentemente do habitual e, em segundo lugar, porque essas dificuldades técnicas podem levar ao deslocamento do cabo-eletrodo e à própria lesão do vaso e do ventrículo direito (VD), podendo gerar riscos ao paciente ${ }^{6}$.

A PVCSE é uma condição rara que não agrega prejuízo clínico ao paciente, na maioria dos casos. Seu diagnóstico é habitualmente incidental, especialmente após a passagem de acesso venoso central, catéteres ou implante de outros dispositivos cardíacos, como, por exemplo, marcapasso. Entretanto, com o aumento da necessidade de procedimentos invasivos torácicos, sua presença pode gerar maior complexidade durante o método, por vezes dificultando a progressão do guia e ocorrendo a chance de lesão das estruturas envolvidas, como já citado. Sua suspeita precoce, por meio de exames complementares previamente realizados (pré-operatório), é de alta valia, uma vez que prepara o profissional para a realização do procedimento, tornando-o mais rápido e seguro ao paciente, e com menor chance de complicações.

O paciente com PVCSE deve ser submetido à investigação complementar, para exclusão de outras anomalias congênitas concomitantes. Apesar da eventual dificuldade técnica que a PVCSE possa causar mesmo ao médico mais experiente, sua presença não contraindica a realização de qualquer procedimento que necessite da utilização das veias que chegam ao coração.

\section{CONTRIBUIÇÃO DOS AUTORES}

Conceitualização, Lourenço A; Metodologia, Lourenço A; Investigação, Lourenço A; Redação - Primeira versão, Lourenço A e Bueno S; Redação - Revisão \& Edição, Lourenço A e Bueno S; Supervisão, Lourenço A.

\section{REFERÊNCIAS}

1. Bragança EOV. Persistência de veia cava superior esquerda e implante de dispositivos cardíacos eletrônicos. Rev LatinoAm Marcapasso Arritmia. 203;26(4):260-70.

2. Altunkas A, Altunkas F. A very rare subgroup of persistent left superior vena cava in an adult: isolated persistent left superior vena cava with absence of the congenital heart diseases. Acta Med Anatolia. 2014;2(1):32-3.

3. Rodríguez-Fernández JA, Almazán-Soo A. Pacemaker lead implant via the persistent left superior vena cava. Arch Cardiol Mex. 2005;75(Suppl 3):S3-106-12.

4. Bernardes MVAA, Kool R, Ludtke IN, Luz MA, Erzinger FL. Veia cava superior esquerda persistente: relato de caso. J Vasc Bras. 2016;15(2)153-7. https://doi. org/10.1590\%2F1677-5449.002815

5. Goyal SK, Punnam SR, Gita V, Ruberg FL. Persistent left superior vena cava: a case report and review of literature. Cardiovasc Ultrasound. 2008;6:50. https://doi. org/10.1186/1476-7120-6-50

6. Biffi M, Massaro G, Diemberger I, Martignani C, Corzani A, Ziacchi M. Cardiac resynchronization therapy in persistent left superior vena cava: can you do it two-leads-only? Heart Rhythm Case Rep. 2017;3(1):30-2. https://doi. org/10.1016\%2Fj.hrcr.2016.08.004 